\title{
Tendências do estado nutricional de crianças no período de 2008 a 2015: dados do Sistema de Vigilância Alimentar e Nutricional (Sisvan)
}

\section{Trends in the nutritional status among children from 2008 to 2015: Food and Nutrition Surveillance System (Sisvan)}

\author{
Naiara Ferraz Moreira' (D), Camilla de Almeida Soares (D), Túlio da Silva Junqueira² (D), \\ Rita de Cassia Bertolo Martins ${ }^{1}$ (D) \\ 'Universidade Federal da Grande Dourados (UFGD) - Dourados (MS), Brasil. \\ 2Universidade Federal de Lavras (UFLA) - Lavras (MG), Brasil.
}

\begin{abstract}
Como citar: Moreira NF, Soares CA, Junqueira TS, Martins RCB. Tendências do estado nutricional de crianças no período de 2008 a 2015: dados do Sistema de Vigilância Alimentar e Nutricional (Sisvan). Cad Saúde Colet, 2020;28(3):447-454. https://doi.org/10.1590/1414-462X202028030133
\end{abstract}

\section{Resumo}

Introdução: O Sistema de Vigilância Alimentar e Nutricional (Sisvan) é de abrangência nacional e essencial para monitorar o crescimento e o desenvolvimento da criança na Atenção Primária à Saúde. Objetivo: Descrever a cobertura e a tendência temporal do estado nutricional de crianças de 5 a 9 anos cadastradas no Sisvan entre 2008 e 2015. Método: Análise de regressão linear simples para verificar a tendência temporal do número de registros, da cobertura e da classificação do estado nutricional de crianças de 5 a 9 anos em quatro territórios (município de Dourados, estado do Mato Grosso do Sul, região Centro-Oeste e Brasil). Resultados: Verificou-se tendência de aumento no número de registros, exceto para Dourados, que apresentou padrão irregular $(p=0,123)$. Magreza e eutrofia apresentaram tendências de queda, enquanto sobrepeso e obesidade aumentaram no período e nos territórios avaliados, com exceção de Dourados, que não apresentou resultados significativos para eutrofia $(p=0,145)$ e obesidade ( $p=0,318)$. Conclusão: O número de registros e, consequentemente, a cobertura do Sisvan aumentaram de forma significativa em três dos quatro territórios avaliados. Os resultados referentes ao estado nutricional retratam e fortalecem o processo de transição nutricional vivenciado no país.

Palavras-chave: criança; vigilância nutricional; estado nutricional; sistemas de informação; estudos de séries temporais.

\begin{abstract}
Background: The Food and Nutrition Surveillance System (Sisvan) is a national and essential System to monitor the growth and development of children in Primary Health Care services. Objective: To describe the coverage and temporal trend of the nutritional status of children aged 5 to 9 registered in Sisvan between 2008 and 2015. Method: Simple linear regression analysis was performed to verify the temporal trend in the number of records, coverage, and nutritional status classification among children from 5 to 9 years old in four territories (Dourados, Mato Grosso do Sul, Midwest region and Brazil). Results: There was a rising trend in the number of records, except for Dourados that presented irregular patterns $(p=0.123)$. Underweight and normal weight presented falling trends, while overweight and
\end{abstract}

Este é um artigo publicado em acesso aberto (Open Access) sob a licença Creative Commons Attribution, que permite uso, distribuição e reprodução em qualquer meio, sem restrições desde que o trabalho original seja corretamente citado.

\footnotetext{
Trabalho realizado utilizando dados nacionais, regionais, estaduais e locais obtidos da plataforma do Sistema de Vigilância Alimentar e Nutricional (SISVAN) - Dourados (MS), Brasil.

Correspondência: Naiara Ferraz Moreira. E-mail: naiaraferraz@ymail.com

Fonte de financiamento: Para a realização do presente manuscrito não houve fonte de financiamento. Foram utilizados dados disponibilizados na base de dados do Sistema de Vigilância Alimentar e Nutricional (SISVAN).

Conflito de interesses: nada a declarar.

Recebido em: Jun. 11, 2017. Aprovado em: Out. 07, 2019
} 
obesity increased in the period in the territories evaluated, except for Dourados, which did not present significant results for normal weight $(p=0.145)$ and obesity $(p=0,318)$. Conclusion: The number of records and, consequently, the coverage of Sisvan increased significantly in three territories evaluated in the period. Results of the nutritional status strengthen the nutritional transition process experienced in Brazil.

Keywords: child; nutritional surveillance; nutritional status; information systems; time serie studies.

\section{INTRODUÇÃO}

O processo de transição nutricional é caracterizado pela mudança do perfil alimentar, como o aumento no consumo de alimentos de alta densidade energética e ultraprocessado $\mathrm{s}^{1-3}$. Esse processo está intimamente associado à transição epidemiológica, que é caracterizada pela redução das prevalências de doenças infecciosas e parasitárias e pelo aumento das Doenças Crônicas Não Transmissíveis (DCNT) ${ }^{1,3}$. Tais processos refletem a realidade da população brasileira em todas as faixas etárias, inclusive entre as crianças ${ }^{1-3}$.

Essas transformações foram influenciadas por fatores sociais, como a diminuição da pobreza, da fome e da escassez de alimentos, levando à redução da desnutrição, e por mudanças nos fatores ambientais e de estilo de vida que contribuíram para a epidemia da obesidade ${ }^{1,4,5}$.

A obesidade infantil aumenta o risco para o desenvolvimento de outras DCNT durante essa fase da vida, assim como na vida adulta ${ }^{5,6}$. Dessa forma, por meio de intervenções focadas na promoção e recuperação da saúde e na prevenção de agravos nessa faixa etária, pode-se elevar a qualidade de vida durante a infância e reduzir o risco para o desenvolvimento de outras doenças a curto e longo prazo 7 .

Nesse contexto, a Vigilância Alimentar e Nutricional (VAN) apresenta papel fundamental na vigilância em saúde, pois objetiva avaliar e monitorar o estado nutricional e o consumo alimentar da população de forma contínua e permanente para o planejamento de ações voltadas à promoção à saúde e para o diagnóstico de segurança alimentar e nutricional da população' ${ }^{1}$. Nesse sentido, a VAN contribui para a organização da atenção nutricional e a definição de prioridades no atendimento à população, além de promover práticas adequadas ao enfrentamento dos problemas existentes, devendo estar inserida no cotidiano das equipes de atenção básica ${ }^{1,6}$.

Para monitorar o diagnóstico alimentar e nutricional da população atendida pelo SUS, foi criado o Sistema de Vigilância Alimentar e Nutricional (Sisvan) na década de 1990, com o objetivo de identificar agravos e riscos relacionados a aspectos alimentares e nutricionais de indivíduos ou grupos, segundo território e/ou faixa etária. Assim, o Sisvan fornece informações importantes que servem de subsídio para o planejamento, a organização e o cuidado com a população, bem como para a formulação e a atualização de políticas públicas na área da saúde ${ }^{1,6,8}$.

A expansão das ações de VAN teve início a partir do ano de 2004, quando o Sisvan começou a ser utilizado para o acompanhamento do estado nutricional da população em todas as fases de vida. Com o intuito de melhorar o sistema e suas funções, foi lançado em 2008 o Sisvan WEB, versão on-line do sistema, com inclusão de curvas de crescimento da Organização Mundial da Saúde (OMS) e inserção de ferramentas para a avaliação do consumo alimentar ${ }^{6,9,10}$.

Estudos realizados com diferentes faixas etárias têm apresentado dados registrados no Sisvan ${ }^{11-18}$ e discutido sobre eles. Assim, com base na importância desses dados para o diagnóstico alimentar e nutricional da população, o objetivo do presente trabalho foi descrever a cobertura do Sisvan e a variação temporal do estado nutricional de crianças de 5 a 9 anos, que foram cadastradas no Sisvan no período de 2008 a 2015, no Brasil, na região Centro-Oeste, no estado do Mato Grosso do Sul (MS) e na cidade de Dourados.

\section{MÉTODO}

Trata-se de um estudo descritivo realizado com dados secundários obtidos no mês de agosto de 2016, do Sistema de Informação do Departamento de Atenção Básica (DAB), por meio do endereço eletrônico: http://dabsistemas.saude.gov.br/sistemas/. Entre os sistemas do DAB disponíveis na Plataforma on-line selecionou-se o Sisvan, e, então, pôde-se acessar os relatórios públicos referentes aos anos de 2008 a 2015 . O ano de 2008 foi escolhido para início 
do estudo, pois nesse ano o Sistema passou a ser informatizado (Sisvan WEB), e os relatórios, disponibilizados publicamente.

Para obtenção dos dados referentes ao estado nutricional, selecionaram-se, no módulo gerador de relatórios, os seguintes itens para a busca: tipo de relatório - estado nutricional dos indivíduos acompanhados; ano de referência - 2008; mês de referência - todos; agrupado por - município (então se acrescentaram a região Centro-Oeste, o estado de MS e o município de Dourados); região de cobertura - todas; fase da vida - criança; idade - de 5 anos a menos de 10 anos completos; índice antropométrico - IMC para idade; sexo - todos; raça/cor - todas; acompanhamentos registrados no Sisvan - todos; povos e comunidades - todos; escolaridade - todos; tipo de relatório - exportar para Microsoft Excel.

Após a seleção de todos os itens citados, selecionou-se o botão "visualizar", e, então, o relatório dos dados agregados referentes ao estado nutricional das crianças acompanhadas, segundo o IMC para idade, foi apresentado em formato de tabela no Microsoft Excel. Posteriormente, repetiu-se a busca utilizando os mesmos critérios para os anos de 2009 a 2015. As tabelas continham o número de registros e o percentual para cada classificação do estado nutricional.

O IMC para idade foi selecionado para efetuar a busca no Sisvan, pois, além de ser utilizado para avaliar o estado nutricional de crianças, permite a continuidade nas demais fases de vida ${ }^{10}$.

Por meio do modelo de regressão linear, analisou-se a tendência de variação temporal, conforme análise realizada pelo estudo de Vigilância de Fatores de Risco e Proteção para Doenças Crônicas por Inquérito Telefônico (Vigitel) ${ }^{19}$. Consideraram-se como variáveis de desfecho o número de registros e a prevalência de cada categoria do estado nutricional, e como variável explanatória o ano do levantamento de dados (2008 a 2015). Todas as análises foram realizadas para cada um dos quatro territórios. Valores de coeficiente de regressão linear e seus respectivos valores de $p$ foram apresentados. Considerou-se significância estatística quando os valores de $p$ foram iguais ou inferiores a 0,05 . Utilizou-se do programa estatístico SPSS (versão 19) para a referida análise.

A cobertura dos registros do Sisvan para o grupo de crianças de 5 a 9 anos foi calculada em percentual para os quatro territórios, considerando o número de crianças cadastradas no Sisvan e a população da mesma idade de acordo com o censo demográfico de $2010^{20}$.

\section{RESULTADOS}

Por meio das análises dos relatórios públicos do Sisvan, referentes ao estado nutricional de crianças de 5 a 9 anos no período de 2008 a 2015, identificou-se tendência significativa de variação temporal do número de registros, exceto para o município de Dourados, e do estado nutricional nos quatro territórios avaliados.

O número de registro no Sisvan evoluiu de modo favorável, apresentando tendência de aumento no estado de MS, na região Centro-Oeste e no Brasil. No MS, a variação anual média foi de $4.228(p<0,001)$. Na região Centro-Oeste, observou-se aumento anual

Tabela 1. Tendência temporal do número de registros do estado nutricional de crianças de 5 a 9 anos no Sisvan, Dourados, Mato Grosso do Sul, Centro-Oeste, Brasil, 2008-2015

\begin{tabular}{|c|c|c|c|c|c|c|c|c|c|c|c|}
\hline \multirow{2}{*}{ Território } & \multicolumn{8}{|c|}{ Ano de avaliação } & \multirow{2}{*}{$\begin{array}{c}\text { Variação } \\
\text { anual } \\
\text { média } \\
(n)^{* * *}\end{array}$} & \multirow{2}{*}{$\begin{array}{l}\text { Valor } \\
\text { de } p\end{array}$} & \multirow{2}{*}{ Tendência } \\
\hline & 2008 & 2009 & 2010 & 2011 & 2012 & 2013 & 2014 & 2015 & & & \\
\hline Dourados & 2.436 & 1.423 & 1.550 & 1.738 & 3.685 & 3.424 & 2.464 & 3.126 & 210 & 0,123 & - \\
\hline $\mathrm{MS}^{*}$ & 21.375 & 20.859 & 23.550 & 27.625 & 33.110 & 37.228 & 41.258 & 50.894 & 4.228 & $<0,001$ & Aumento \\
\hline $\mathrm{CO}^{* *}$ & 101.339 & 103.603 & 120.017 & 130.328 & 137.362 & 170.041 & 194.867 & 244.437 & 19.228 & $<0,001$ & Aumento \\
\hline Brasil & 2.042 .830 & 1.976 .629 & 2.158 .609 & 2.173 .361 & 2.331 .304 & 3.027 .004 & 3.469 .049 & 4.025 .308 & 286.935 & 0,001 & Aumento \\
\hline
\end{tabular}

*MS = Mato Grosso do Sul; **CO = região Centro-Oeste. Fonte: SISVAN web 2008-2015; ***Número de avaliações. 
médio de 19.228 ( $\mathrm{p}<0,001)$. No Brasil, a análise de tendência demostrou aumento anual de 286.935 registros $(p=0,001)$ (Tabela 1$)$. Ao analisar os dados referentes aos registros do município de Dourados, nota-se que não houve significância estatística $(p=0,123)$, provavelmente por causa do perfil irregular de registros no período, ora marcado por aumento, ora por quedas constantes (Tabela 1).

Tomando por base os dados do último censo demográfico realizado pelo IBGE, em 201020, e fazendo um comparativo para o mesmo ano com os dados de registro do Sisvan nos quatro territórios, a cobertura do Sisvan em Dourados, na faixa etária de 5 a 9 anos de idade, foi de $10 \%$ ( 1.550 crianças de um total de 15.307 tiveram dados registrados no Sisvan). No MS, o percentual de crianças avaliadas com registro no Sisvan foi de quase $12 \%$ ( 23.550 registros de um total de 196.829). Os dados da região Centro-Oeste seguem na mesma direção, com $11 \%$ de registros no Sisvan em 2010 (120.017 registros de um total de 1.125 .369 crianças). Dados nacionais revelaram cobertura de $14 \%$ do total de crianças brasileiras (2.158.609 registros de um total de 14.969.375 crianças) no mesmo ano de realização do censo. Adicionalmente, analisou-se a tendência temporal da cobertura do Sisvan nos quatro territórios, tomando o censo de 2010 como referência. Observou-se que a cobertura do Sisvan aumentou no MS ( $2,15 \mathrm{pp} ; \mathrm{p}<0,001)$, na região Centro-Oeste $(1,71 \mathrm{pp} ; \mathrm{p}<0,001)$ e no Brasil $(1,92 \mathrm{pp} ; \mathrm{p}=0,001)$ no período de oito anos. Dados do município de Dourados não mostraram tendência significativa (Tabela 2).

Tabela 2. Prevalência (\%) e tendência temporal da cobertura do Sisvan referente aos dados de crianças de 5 a 9 anos, Dourados, Mato Grosso do Sul, Centro-Oeste, Brasil, 2008-2015

\begin{tabular}{|c|c|c|c|c|c|c|c|c|c|c|c|c|}
\hline \multirow{2}{*}{ Território } & \multirow{2}{*}{$\begin{array}{c}\text { Total de } \\
\text { crianças*** }^{*}\end{array}$} & \multicolumn{8}{|c|}{ Ano de avaliação } & \multirow{2}{*}{$\begin{array}{c}\text { Variação } \\
\text { anual } \\
\text { média } \\
\text { (pp) }\end{array}$} & \multirow{2}{*}{$\begin{array}{l}\text { Valor } \\
\text { de } p\end{array}$} & \multirow{2}{*}{ Tendência } \\
\hline & & 2008 & 2009 & 2010 & 2011 & 2012 & 2013 & 2014 & 2015 & & & \\
\hline Dourados & 15.307 & 15,9 & 9,3 & 10,1 & 11,4 & 24,1 & 22,4 & 16,1 & 20,4 & 1,37 & 0,124 & - \\
\hline MS* & 23.550 & 10,9 & 10,6 & 12,0 & 14,0 & 16,8 & 18,9 & 21,0 & 25,9 & 2,15 & $<0,001$ & Aumento \\
\hline $\mathrm{CO}^{* *}$ & 1.125 .369 & 9,0 & 9,2 & 10,7 & 11,6 & 12,2 & 15,1 & 17,3 & 21,7 & 1,71 & $<0,001$ & Aumento \\
\hline
\end{tabular}

*MS = Mato Grosso do Sul; **CO = região Centro-Oeste; ***Dados do censo demográfico do ano de 2010. Fonte: SISVAN web 2008-2015.

Tabela 3. Prevalência (\%) e tendência temporal do estado nutricional de crianças de 5 a 9 anos no Sisvan, Dourados, Mato Grosso do Sul, CentroOeste, Brasil, 2008-2015

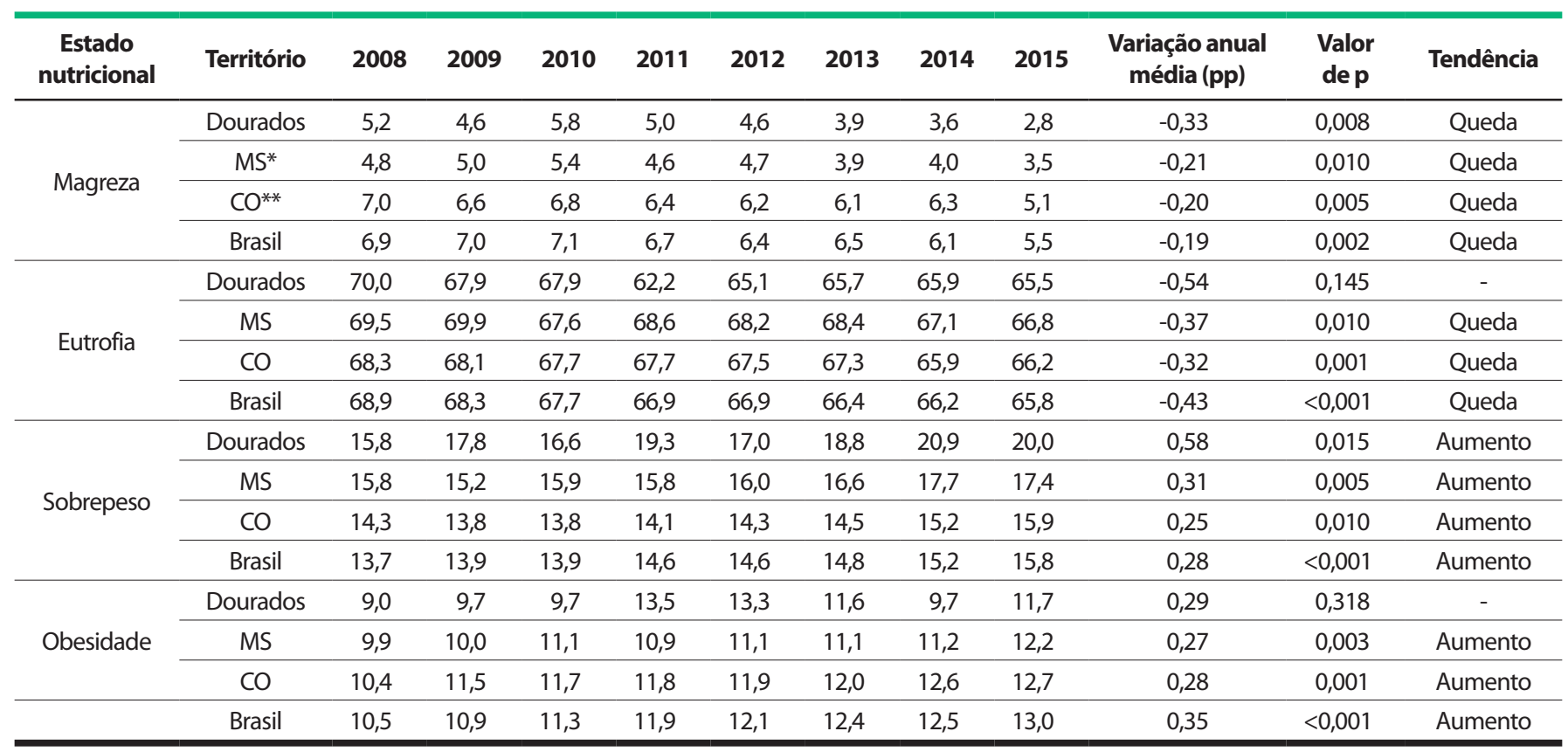

*MS = Mato Grosso do Sul; **CO = região Centro-Oeste. Fonte: SISVAN web 2008-2015. 
Na Tabela 3, pode-se observar a prevalência e a tendência temporal da classificação do estado nutricional das crianças de 5 a 9 anos, nos quatro territórios, no período de 2008 a 2015. A prevalência de magreza apresentou tendência significativa de queda: a redução anual média foi de $0,33 \mathrm{pp}$ em Dourados $(p=0,008), 0,21 \mathrm{pp}$ em MS $(\mathrm{p}=0,010), 0,20 \mathrm{pp}$ no Centro-Oeste $(p=0,005)$ e $0,19 p p$ no Brasil $(p=0,002)$. Em relação à eutrofia, constatou-se queda em três dos quatro territórios avaliados. A redução anual média foi de 0,37 pp no MS $(p=0,010), 0,32$ pp no Centro-Oeste $(p=0,001)$ e de 0,43 pp no Brasil $(p<0,001)$ (Tabela 3).

A prevalência de sobrepeso apresentou tendência de aumento no período nos quatro territórios. Em Dourados, a frequência de crianças com sobrepeso teve aumento médio anual de $0,58 \mathrm{pp}(p=0,015)$. No MS, Centro-Oeste e Brasil, o aumento da prevalência de sobrepeso foi, respectivamente, de $0,31 \mathrm{pp}(\mathrm{p}=0,005), 0,25 \mathrm{pp}(\mathrm{p}=0,01)$ e $0,28 \mathrm{pp}(\mathrm{p}<0,001)$ em média ao ano (Tabela 3). Quanto à obesidade, a prevalência do aumento anual médio foi de 0,27 pp no estado de MS, 0,28 pp na região Centro-Oeste e 0,35 pp no Brasil ao ano $(p \leq 0,001)$. Em Dourados, apesar das flutuações, observou-se variação positiva, não significativa ( $p=0,318$ ) (Tabela 3 ).

\section{DISCUSSÃO}

Neste estudo, verificou-se aumento no número de registros de peso e estatura, consequentemente do IMC/idade, no Sisvan, de crianças de 5 a 9 anos no período de oito anos, na cidade de Dourados, no estado de MS, na região Centro-Oeste e no Brasil. Porém, é importante destacar que o padrão observado na cidade de Dourados apresentou flutuação não linear, com mais declínios e elevações do que os demais territórios avaliados, dados esses confirmados por análises de tendência temporal.

O aumento dos registros ao longo do tempo verificado neste estudo pode ter sido decorrente de avanços nas ações e políticas de alimentação e nutrição do Ministério da Saúde, por exemplo, a elaboração dos Protocolos do Sistema de VAN na assistência à saúde. Nesses protocolos, oferecem-se todas as normas de padronização e atendimentos, que devem ser utilizados em cada estado nutricional, inclusive pelos nutricionistas que trabalham na atenção básica ${ }^{6,10}$.

O Programa Bolsa Família contribuiu para o aumento do número de registros. Trata-se de um programa federal de transferência de renda, criado em 2004, que tem como seu principal objetivo o combate à fome, a promoção do acesso à rede de serviços públicos e a promoção da segurança alimentar e nutricional ${ }^{21}$. Entre as condicionalidades do programa está a realização de VAN de seus beneficiários ${ }^{6,10}$.

Outro programa do governo federal que pode estar relacionado ao aumento nos registros no Sisvan é o Programa Saúde na Escola (PSE). Esse programa, criado em 2007, tem por objetivo desenvolver ações de saúde que devem ser realizadas na rede pública de educação básica, de acordo com os princípios e as diretrizes do SUS, envolvendo, entre outras ações, a avaliação nutricional dos escolares $s^{6,10,22}$. Não foi possível avaliar a influência do PSE sobre os registros do Sisvan, pois os relatórios públicos não disponibilizam tal informação.

A criação do Núcleo de Apoio à Saúde da Família (NASF), atual Núcleo Ampliado de Saúde da Família e Atenção Primária (NASF-AP), teve como objetivo aumentar a resolutividade e a capacidade de resposta das equipes de saúde da família aos problemas da população 23,24 , e pode ter contribuído para o aumento no número de registros. Entre as atribuições do nutricionista do NASF encontra-se o matriciamento das equipes de saúde para a VAN das famílias acompanhadas pela atenção básica à saúde.

Outro ponto que deve ser levado em consideração para o aprimoramento da VAN e, consequentemente, dos registros no Sisvan é o fortalecimento das diretrizes da Política Nacional de Alimentação e Nutrição (PNAN). No ano de 2011, após a PNAN completar 10 anos, ocorreu o processo de atualização e melhoramento de suas bases e diretrizes, sendo a VAN uma delas, enfocadas na atenção nutricional no SUS ${ }^{1,11}$.

Apesar do número de registros ter aumentado no período estudado, ainda é considerado baixo. Estudos realizados em outros estados brasileiros, com dados de crianças de faixa etária variada obtidos por meio do Sisvan, também encontraram baixas frequências de 
registros ${ }^{11,13,14,18,25}$. No Rio Grande do Sul, o percentual de crianças de 0 a 10 anos com dados registrados no Sisvan foi de $10,5 \%$ no ano de $2006^{11}$, e para crianças de 5 a 10 anos foi de $5,1 \%$ em $2010^{18}$. Em São Paulo, no ano de 2010, a região de Ribeirão Preto alcançou cobertura de $12,8 \%$, contrastando com a região de Presidente Prudente, que apresentou $42,6 \%{ }^{13}$. Ainda em São Paulo, outro estudo realizado pelo Departamento Regional de Saúde (DRS) do estado, também no ano de 2010, identificou baixa cobertura de registro de crianças de 0 a 10 anos no Sisvan, apenas 8,1\%, variando de 3,9\% na Regional da Grande São Paulo a 28,6\% na Regional de São José do Rio Preto; no entanto, foi a fase de vida que apresentou maiores percentuais de registro em todas as regionais de saúde ${ }^{25}$. Em Minas Gerais, em estudo com crianças menores de 2 anos na Regional de Saúde de Belo Horizonte, observou-se cobertura de 4,3\% em 2008 a $10,7 \%$ em $2011^{14}$.

Para a realização da avaliação antropométrica e dos cadastrados no Sisvan, muitos aspectos devem ser levados em consideração. Primeiramente, é importante destacar que é fundamental que as unidades de saúde possuam, pelo menos, uma balança e um estadiômetro para menores e para maiores de 2 anos de idade. Contudo, além da realização das aferições que permitem alimentar o Sistema, é de suma importância que os equipamentos antropométricos sejam calibrados e passem por manutenção periódica, pois a má qualidade dos equipamentos pode contribuir para a subestimação ou superestimação dos dados e, consequentemente, interferir na classificação do estado nutricional ${ }^{8,26}$. Além disso, para a correta aferição das medidas antropométricas, os equipamentos devem estar localizados em ambiente reservado e com o espaço adequado para a avaliação ${ }^{26,27}$.

Outro importante fator para a adequada realização da VAN nas unidades de saúde é que a equipe de saúde esteja consciente da importância da VAN, e, para que isso aconteça, é fundamental que as equipes sejam capacitadas ${ }^{26}$. Para a formação em VAN, o Ministério da Saúde recomenda atividades teóricas e práticas que contemplem os temas: importância da VAN no cuidado e na gestão em saúde; método antropométrico; avaliação dos marcadores de consumo alimentar; registro de dados em prontuários, formulários, cadernetas de acompanhamento de saúde e sistemas de informação; e avaliação do estado nutricional individual e coletiva ${ }^{6}$.

Em relação ao estado nutricional das crianças nos territórios avaliados, apesar do baixo percentual de registro, pode-se observar que os resultados encontrados seguem o observado em pesquisas de representatividade nacional, como a Pesquisa de Orçamentos Familiares (POF, 2008-2009) ${ }^{28}$. Nota-se baixa frequência de magreza em todos os anos avaliados, assim como observado na POF $(4,1 \%)^{28}$. Destaca-se também a importante queda nos percentuais de magreza ao observar os resultados da tendência temporal, corroborando outros resultados que evidenciam o processo de transição nutricional vivido em nosso país ${ }^{28}$.

Em relação ao sobrepeso, constataram-se prevalências elevadas neste estudo e com tendência temporal de aumento para todos os territórios avaliados. Cabe destacar que as prevalências de obesidade encontradas foram consideradas elevadas nos quatro territórios, atingindo, em 2015, 11,7\% em Dourados, 12,2\% no MS, 12,7\% no Centro-Oeste e 13\% no Brasil, com aumento anual médio significativo no período, exceto para Dourados. Dados da POF apontam que cerca de $1 / 3$ das crianças de 5 a 9 anos apresentava excesso de peso (sobrepeso e obesidade) no Brasil, e, na região Centro-Oeste, $37,9 \%$ dos meninos e $32,4 \%$ das meninas encontravam-se nessa condição nutricional. Os resultados referentes à obesidade preocupam mais ainda, visto que, em uma população saudável, espera-se que esse percentual não ultrapasse $2,3 \%{ }^{27}$.

Em razão do desenho deste estudo, não se avaliaram os fatores associados a esses registros, visto que não é possível identificar as características pessoais dos indivíduos avaliados, como características demográficas, socioeconômicas e de estilo de vida. Acredita-se que o padrão irregular do número de registros na cidade de Dourados possa ter refletido nos resultados não significativos de queda na prevalência de eutrofia e aumento na prevalência de obesidade, diferindo do observado nos demais territórios avaliados.

Contudo, avaliaram-se a cobertura e a variação dos registros no Sisvan de crianças de 5 a 9 anos e do estado nutricional nos quatro territórios. Conclui-se que houve tendência de aumento na cobertura do Sisvan e no percentual de excesso de peso e tendência de queda no percentual de magreza e eutrofia no estado do MS, na região Centro-Oeste e no 
Brasil. Para melhorar o percentual e a qualidade dos registros no Sisvan, além dos fatores anteriormente citados, sugere-se estabelecer vínculo entre universidades e secretárias de saúde. A universidade poderia contribuir para a realização de levantamento da estrutura, dificuldades encontradas pelos profissionais de saúde, capacitação para equipes de saúde, análise e divulgação das informações, além proporcionar aos acadêmicos a oportunidade de vivenciar a prática do serviço, o que pode contribuir para a formação de profissionais mais conscientes do seu papel.

\section{REFERÊNCIAS}

1. Brasil. Ministério da Saúde. Secretaria de Atenção à Saúde. Departamento de Atenção Básica. Política Nacional de Alimentação e Nutrição. Brasília; 2012. 84 p. (Série B. Textos Básicos de Saúde).

2. Moreira MCN, Gomes R, Sá MRC. Doenças crônicas em crianças e adolescentes: uma revisão bibliográfica. Cien Saude Colet. 2014;19(7):2083-94. http://dx.doi.org/10.1590/1413-81232014197.20122013.

3. Leão $M$, editor. $O$ direito humano à alimentação adequada e o sistema nacional de segurança alimentar e nutricional. Brasília: ABRANDH; 2013.

4. Silva DAS. Sobrepeso e obesidade em crianças de cinco a dez anos de idade beneficiárias do Programa Bolsa Família no estado de Sergipe, Brasil. Rev Paul Pediatr. 2011;29(4):529-35. http://dx.doi.org/10.1590/ S0103-05822011000400010.

5. Azambuja AP, Netto-Oliveira ER, Oliveira AA, Azambuja MA, Rinaldi W. Prevalência de sobrepeso/obesidade e nível econômico de escolares. Rev Paul Pediatr. 2013;31(2):166-71. http://dx.doi.org/10.1590/S010305822013000200006. PMid:23828052.

6. Brasil. Ministério da Saúde. Secretaria de Atenção à Saúde. Departamento de Atenção Básica. Marco de referência da vigilância alimentar e nutricional na atenção básica. Brasília: Ministério da Saúde; 2015.

7. Brasil. Ministério da Saúde. Secretaria de Vigilância em Saúde. Departamento de Análise de Situação de Saúde. Plano de ações estratégicas para o enfrentamento das doenças crônicas não transmissíveis (DCNT) no Brasil 2011-2022. Brasília: Ministério da Saúde; 2011.

8. Fagundes AA, Coitinho D. Vigilância alimentar e nutricional - Sisvan: orientações básicas para a coleta, processamento, análise de dados e informação em serviços de saúde. Brasília: Ministério da Saúde, 2004.

9. Brasil. Ministério da Saúde. Secretaria de Atenção à Saúde. Departamento de Atenção Básica. Indicadores de Vigilância Alimentar e Nutricional: Brasil 2006. Brasília: Ministério da Saúde; 2009.

10. Brasil. Ministério da Saúde. Secretaria de Atenção à Saúde. Departamento de Atenção Básica. Protocolos do Sistema de Vigilância Alimentar e Nutricional - SISVAN na assistência à saúde. Brasília: Ministério da Saúde; 2008.

11. Damé PKV, Pedroso MRO, Marinho CL, Gonçalves VM, Duncan BB, Fisher PD, et al. Sistema de Vigilância Alimentar e Nutricional (Sisvan) em crianças do Rio Grande do Sul, Brasil: cobertura, estado nutricional e confiabilidade dos dados. Cad Saude Publica. 2011;27(11):2155-65. http://dx.doi.org/10.1590/S0102311X2011001100009. PMid:22124493.

12. Venâncio SI, Levy RB, Saldiva SRDM, Mondini L, Stefanini MLR. Sistema de vigilância alimentar e nutricional no Estado de São Paulo, Brasil: experiência da implementação e avaliação do estado nutricional de crianças. Rev Bras Saúde Mater Infant. 2007;7(2):213-20. http://dx.doi.org/10.1590/S1519-38292007000200012.

13. Enes CC, Loiola H, Oliveira MRM. Cobertura populacional do Sistema de Vigilância Alimentar e Nutricional no Estado de São Paulo, Brasil. Cien Saude Colet. 2014;19(5):1543-51. http://dx.doi.org/10.1590/141381232014195.05872013.

14. Ferreira CS, Cherchiglia ML, Cesar CC. O Sistema de Vigilância Alimentar e Nutricional como instrumento de monitoramento da Estratégia Nacional para Alimentação Complementar Saudável. Rev Bras Saúde Mater Infant. 2013;13(2):167-77. http://dx.doi.org/10.1590/S1519-38292013000200010.

15. Coutinho JG, Cardoso AJC, Toral N, Silva ACF, Ubarana JA, Aquino KKNC, et al. A organização da Vigilância Alimentar e Nutricional no Sistema Único de Saúde: histórico e desafios atuais. Rev Bras Epidemiol 2009;12(4):688-99. http://dx.doi.org/10.1590/S1415-790X2009000400018.

16. Rolim MD, Lima SML, Barros DC, Andrade CLT. Avaliação do SISVAN na gestão de ações de alimentação e nutrição em Minas Gerais, Brasil. Cien Saude Colet. 2015;20(8):2359-69. http://dx.doi.org/10.1590/141381232015208.00902015.

17. Felisbino-Mendes MS, Campos MD, Lana FCF. Avaliação do estado nutricional de crianças menores de 10 anos no município de Ferros, Minas Gerais. Rev. Esc. Enferm. 2010;44(2):257-65. http://dx.doi.org/10.1590/ S0080-62342010000200003. 
18. Jung NM, Bairros FS, Neutzling MB. Utilização e cobertura do Sistema de Vigilância Alimentar e Nutricional no Estado do Rio Grande do Sul, Brasil. Cien Saude Colet. 2014;19(5):1379-88. http://dx.doi. org/10.1590/1413-81232014195.20002013.

19. Brasil. Ministério da Saúde. Secretaria de Vigilância em Saúde. Departamento de Vigilância de Doenças e Agravos não Transmissíveis e Promoção da Saúde. Vigitel Brasil 2016: vigilância de fatores de risco e proteção para doenças crônicas por inquérito telefônico: estimativas sobre frequência e distribuição sociodemográfica de fatores de risco e proteção para doenças crônicas nas capitais dos 26 estados brasileiros e no Distrito Federal em 2016. Brasília: Ministério da Saúde; 2017.

20. Instituto Brasileiro de Geografia e Estatística. Indicadores Sociais Municipais: uma análise dos resultados do universo do Censo Demográfico 2010. Brasília: Ministério do Planejamento, Orçamento e Gestão; 2011.

21. Brasil. Ministério da Saúde. Secretaria de Atenção à Saúde. Departamento de Atenção Básica. Manual de orientações sobre o Bolsa Família na Saúde. 3. ed. Brasília: Ministério da Saúde; 2010.

22. Brasil. Ministério da Saúde. Ministério da Educação. Programa Saúde na Escola 2014: passo a passo para adesão. Brasília: Ministério da Saúde; 2014.

23. Brasil. Ministério da Saúde. Núcleo de Apoio à Saúde da Família. Brasília: Ministério da Saúde; 2014. (vol. 1).

24. Brasil. Ministério da Saúde. Portaria no. 2.436, de 21 de setembro de 2017. Aprova a Política Nacional de Atenção Básica, estabelecendo a revisão de diretrizes para a organização da Atenção Básica, no âmbito do Sistema Único de Saúde (SUS). Diário Oficial da União, Brasília, 22 de setembro de 2017; Seção 1.

25. Perez AIDLC, Oliveira TZD, Moraes MAD, Shirassu MM, Ribeiro AB, Coria S. Monitoramento do estado nutricional de usuários de Unidades Básicas de Saúde no Estado de São Paulo por meio do Sistema de Vigilância Alimentar e Nutricional (SISVAN). BEPA. Boletim Epidemiológico. 2013;10(116):1-13.

26. Bagni UV, Barros DC. . Capacitação em antropometria como base para o fortalecimento do Sistema de Vigilância Alimentar e Nutricional no Brasil. Rev Nutr. 2012;25(3):393-402. http://dx.doi.org/10.1590/ S1415-52732012000300009.

27. Brasil. Ministério da Saúde. Secretaria de Atenção à Saúde. Departamento de Atenção Básica. Orientações para a coleta e análise de dados antropométricos em serviços de saúde: Norma Técnica do Sistema de Vigilância Alimentar e Nutricional - SISVAN. Brasília: Ministério da Saúde, 2011.

28. Instituto Brasileiro de Geografia e Estatística. Pesquisa de Orçamentos Familiares 2008-2009. Rio de Janeiro: Instituto Brasileiro de Geografia e Estatística; 2010. 